OPEN ACCESS

Edited by:

Ellis Meng,

University of Southern California,

United States

Reviewed by:

Patrick Ruther,

Institut für Mikrosystemtechnik, Albert Ludwigs Universität Freiburg,

Germany

Chingfu Tsou,

Feng Chia University, Taiwan

${ }^{*}$ Correspondence:

Wasif Khan

khanwasi@msu.edu

Specialty section

This article was submitted to

Micro- and Nanoelectromechanical

Systems,

a section of the journa

Frontiers in Mechanical Engineering

Received: 01 August 2018

Accepted: 19 October 2018

Published: 09 November 2018

Citation:

Khan W, Setien M, Purcell E and Li W (2018) Micro-Reflector Integrated Multichannel $\mu L E D$ Optogenetic Neurostimulator With Enhanced Intensity. Front. Mech. Eng. 4:17. doi: 10.3389/fmech.2018.00017

\section{Micro-Reflector Integrated Multichannel $\mu$ LED Optogenetic Neurostimulator With Enhanced Intensity}

\author{
Wasif Khan ${ }^{1 *}$, Monica Setien ${ }^{2}$, Erin Purcell ${ }^{1,2}$ and Wen $\mathrm{Li}^{1}$ \\ 'Microtechnology Laboratory, Department of Electrical and Computer Engineering, Michigan State University, East Lansing, \\ MI, United States, ${ }^{2}$ Regenerative Electrode Interface Laboratory, Department of Biomedical Engineering, Michigan State \\ University, East Lansing, MI, United States
}

This paper reports the fabrication and characterization of an optical neuro-stimulator array that consists of 32-channel microscale light-emitting diodes ( $\mu$-LEDs) coupled with microscale reflectors for intensity enhancement. The hemi-spherical micro-reflector is able to collect the rear side emission of LED while also acting as a collimator to focus the diverged LED light, aiming toward driving power minimization through light intensity increase, for wireless neuro-stimulator applications. The micro-reflector was constructed by wet etching of silicon followed by aluminum coating as the reflective mirror. The reflective cavity was filled with polydimethylsiloxane (PDMS) that acts as the planarization polymer to facilitate device integration with the $\mu$-LED chip. Deviation of hemi-spherical geometry cavities due to the uneven lateral and vertical etching rate was shown, and the surface morphology was characterized experimentally. Optical intensity enhancement was studied in both simulation and experiments, demonstrating that the micro-reflector enables 65\% intensity enhancement. The reflector-coupled LED had an operating temperature increase of $<1^{\circ} \mathrm{C}$, well within the ANSI/AAMI safety limit for biomedical implants. The potential of the stimulator for use in optogenetics was validated by in vitro experiments.

Keywords: micro-reflector, light emitting diode, silicon wet etching, optogenetics, pixel density

\section{INTRODUCTION}

Optogenetics is the technology of delivering light to tissues of interest while collecting readouts from the cells using targeted control tools (Deisseroth, 2011). Optogenetic applications could range toward restoring motor control in order to treat spinal cord injury and stroke, or other neurological disorders, such as patients with Parkinson's disease and depression (Fan et al., 2014). Research in the field of optogenetics spans primarily in two branches, the development of the light-sensitive opsins that is used to genetically modify the neurons and the development of the brain-machine interface, that includes stimulation sources, waveguides for precise and in-depth light delivery and could also include recording electrodes for simultaneous light-evoked signal capture. The targeted cells, after being transfected by the appropriate opsin, requires optical stimulation of sufficient intensity [1 $\mathrm{mW} / \mathrm{mm}^{2}$ or $7 \mathrm{~mW} / \mathrm{mm}^{2}$ for excitatory and inhibitory opsins (Aravanis et al., 2007), respectively] to activate or silence the cell. One major challenge in developing optogenetic tools is the need to 
express high levels of the opsins due to the relatively small optical current mediated by each opsin molecule (Aravanis et al., 2007; Deisseroth, 2011). Moreover, opsin activation largely depends on the wavelength and the intensity of the input light source. A successful optogenetic tool requires a structured, timevarying light stimulus of a certain minimum intensity that could be automatically modulated based on the difference between desired and measured outputs (Grosenick et al., 2015). Various types of light delivery tools have been reported with enhanced performance and extended functionalities. Examples include microscope focused light delivery (Ayling et al., 2009), SiON 3D waveguides (Zorzos et al., 2012), polymer waveguides (Kwon and Li, 2013; Wu et al., 2013), glass optrode-array (Abaya et al., 2012), and fiber-optic probes (Stark et al., 2012; Chen et al., 2013; Nussinovitch and Gepstein, 2015).

During the early stage, the activation of opsins was most commonly accomplished by illuminating with a laser, laser diode, or light-emitting-diode (LED) coupled with optical fibers (Campagnola et al., 2008; Han et al., 2009; Zhang et al., 2010). Advantages of using optical fibers include coherence with narrow bandwidth accompanied by a low optical power loss through direct illumination. Simultaneous coupling of peripheral tools incorporated with mature technology is an added advantage. While these techniques enable a strong illumination with high intensity, they fail to provide a considerable spatial resolution resulting in the inability of multi-site stimulation. Most importantly, fibers coupled with external optical sources significantly compromise the natural behavior of the subjects. In order to eliminate these difficulties, microscale LEDs ( $\mu$ LEDs) have been widely used as the light stimulus source (Grossman et al., 2010; McGovern et al., 2010; Tokuda et al., 2012; McAlinden et al., 2015), which is more compact, inexpensive, and power efficient than lasers or laser diodes. Furthermore, $\mu$-LEDs can be integrated with wireless interfaces (Montgomery et al., 2015; Park et al., 2015), such as batteries (Gagnon-Turcotte et al., 2017) or utilizing electromagnetic near-field (Shin et al., 2017; Aldaoud et al., 2018; Biswas et al., 2018; Khan et al., 2018) and far-field region (Kim et al., 2013; Park et al., 2015) for single and multi-site in-vivo stimulation in freely moving subjects. Simultaneously, wireless data links, such as Bluetooth, can also be integrated with $\mu$-LEDs to achieve bi-directional interfaces with the nervous systems (Jeong et al., 2015).

Although providing significant benefits over the optic fiber based stimulation systems, $\mu$-LED stimulation possesses undesirable drawbacks toward an effective stimulation system. Being a low power setup, $\mu$-LEDs have a limited penetration depth compared with the high power fiber optic coupled setups. In addition, light absorption and light scattering within the brain tissue results in poor spatial resolution. Studies performed by other groups indicated intensity reduction to $10 \%$ of the threshold value only within a few hundred micrometers of the tissue surface (Adamantidis et al., 2007; Yizhar et al., 2011), this phenomena being particularly problematic for blue lights compared to red and infrared emission that have better penetration within tissues. While considering the LED coupled micro-optic fiber setups, radiation loss occurs due to the low out-coupling efficiency between the LED and the fiber. This phenomenon generates from the LED's wide radiation angle and the Lambertian emission pattern (Bi et al., 2016). Moreover, insertion of LED coupled fibers into the brain tissues requires more invasive surgeries compared to an epidural stimulator that is placed over the cortical surface.

A low power system is desired toward optimizing untethered stimulation systems, especially for inductively coupled wireless power transfer mechanisms aimed for behavioral studies. The $\mu$ LED based stimulation interface is an attractive alternative in this case; however, these setups have limited available energy due to the low power transfer efficiency from the inductive coupling. Therefore, it becomes very crucial to effectively utilize the light generated from this limited energy.

To address the need for intensity boost from $\mu$-LEDs while achieving a better spatial resolution toward multi-site stimulation, we propose a reflector-coupled $\mu$-LED array to act as a surface light source for minimally invasive, epidural optogenetic neuromodulation. Wafer-level microfabrication and assembly methods were developed to construct the proposed array in a fast and reliable manner. The rest of the paper is structured as follows: section Methods discusses the methods of the fabrication process and the simulation, bench top and in vitro experimental procedures, and section Results and Discussion consists of the discussions on the aforementioned experiments and outcomes.

\section{METHODS}

\section{Device Design}

As illustrated in Figure 1, the proposed stimulator consists of an array of 32 stimulation channels distributed in two symmetric panels, with $4 \times 4$ channels on each panel. Each of these stimulation channels has a $\mu$-LED coupled with a reflective silicon cavity (Khan and $\mathrm{Li}, 2017$ ). The $\mu$-LED (CREE TR2227tm) has a surface area of $270 \times 220 \mu \mathrm{m}$ and a thickness of $50 \mu \mathrm{m}$, and the central illumination wavelength of $465 \mathrm{~nm}$ for excitation of optogenetic opsins. The diameter of each hemispherical reflective cavity is $300 \mu \mathrm{m}$ and the distance between adjacent cavities is $\sim 430 \mu \mathrm{m}$. The overall dimension of each panel is $3 \times 3 \mathrm{~mm}$, which is intended to cover one hemisphere of the primary visual cortex (V1) of rats. The two-panel design exclusively allows stimulation of both cortices individually. To reduce the number of interconnects to control circuitry, LEDs on each panel share a single ground wire while having separate power wires to enable individual control of one or more LEDs at a time.

\section{Device Fabrication}

Figure 2 illustrates a simplified process flow for device fabrication, which includes the following major steps.

\section{Wafer Preparation for Silicon Etching}

Silicon wafers with $300 \mathrm{~nm}$ standard low-pressure chemical vapor deposited (LPCVD) nitride were purchased from University Wafers, Boston, MA, USA. Fabrication initiated by cleaning the wafers in acetone and isopropyl alcohol (IPA), followed by deionized (DI) water rinse. Post-cleaning, a layer of 4/500 nm 


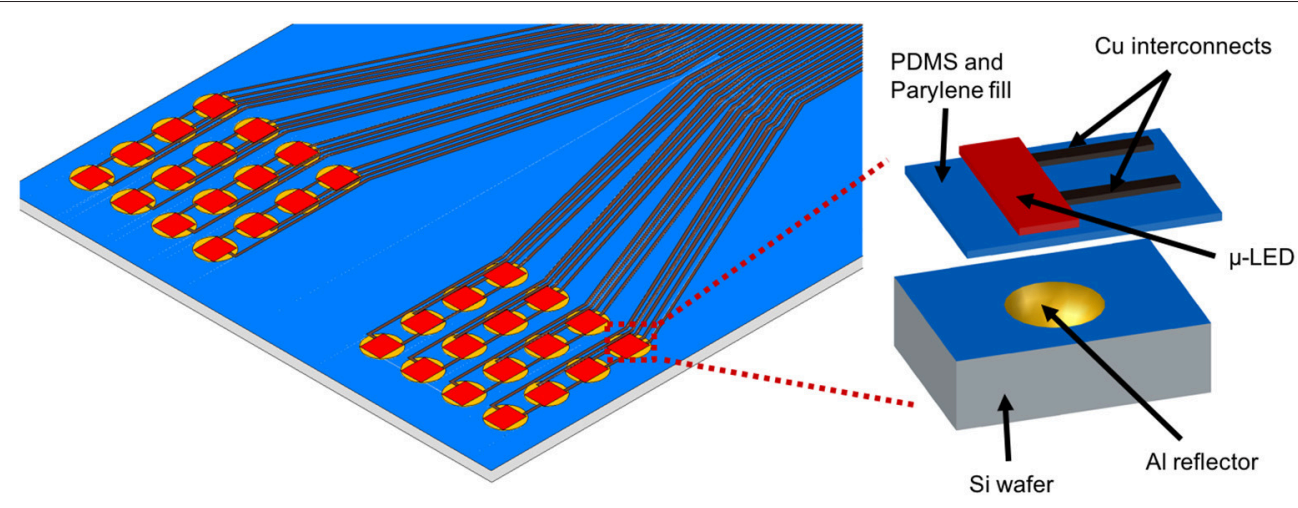

FIGURE 1 | Conceptual schematic of the reflector coupled $\mu$-LED array stimulator.

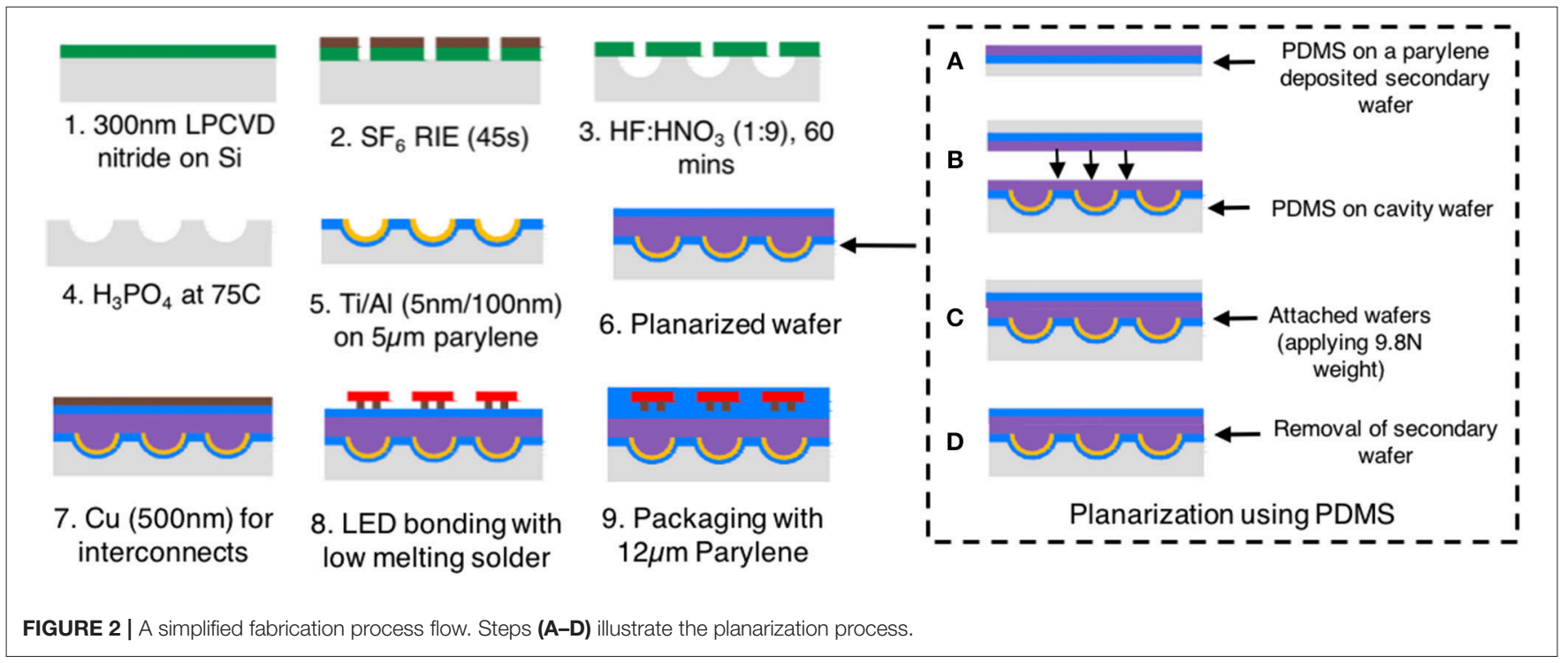

titanium (Ti)/copper $(\mathrm{Cu})$ was thermally evaporated (Auto306, Edwards) and chemically patterned using a photoresist mask. $\mathrm{Ti}$ was deposited as an adhesion layer and $\mathrm{Cu}$ acted as a mask for nitride etching because of its low etching rate in $\mathrm{SF}_{6}$ plasma (Williams et al., 2003). A positive photoresist mask (Shipley S1813, MICROCHEM Corp.) was patterned using ultraviolet (UV) lithography. The unwanted $\mathrm{Cu}$ and $\mathrm{Ti}$ were removed chemically using $\mathrm{Cu}$ etchant (ferric chloride, MG chemicals) and hydrofluoric acid (HF), respectively, followed by DI water rinse. After that, the LPCVD nitride was patterned in a reactive ion etcher (RIE 1701, Nordson March) using $20 \mathrm{sccm} \mathrm{SF}_{6}$ plasma at $250 \mathrm{~W}$ RF power for $45 \mathrm{~s}$. After the reactive etching, the $\mathrm{Cu}$ and Ti used for masking were completely removed using the process mentioned above.

\section{Silicon Etching}

The formation of hemi-spherical cavities utilizing chemical etching is achieved by a mixture of hydrofluoric and nitric acid, $\mathrm{HF}: \mathrm{HNO}_{3}$. Based on the solution recipe reported by Albero et al. (2009), in this paper, we studied several different combinations of masking materials to determine the best protocol for wet silicon etching, including plasma enhanced chemical vapor deposition (PECVD) silicon oxide ( $\mathrm{SiO}$ ), PECVD nitride and LPCVD nitride. For the samples, PECVD nitride was deposited at $300^{\circ} \mathrm{C}$ on $375 \mu \mathrm{m}$ thick silicon wafers using an Oxford PlasmaLab 80 Plus PECVD. It was observed that for a PECVD nitride coated silicon wafer, the nitride layer was rapidly stripped in an $\mathrm{HF}: \mathrm{HNO}_{3}$ (1:9 by volume) solution. An addition of acetic acid, to create a solution of $\mathrm{HF}: \mathrm{HNO}_{3}: \mathrm{CH}_{3} \mathrm{COOH}$ (1:9:1 by volume) did not retard the etching rate of the PECVD nitride. A single layer of $\mathrm{SiO}(100 \mathrm{~nm})$ and a stacked layer of PECVD nitride $(100-300 \mathrm{~nm})$ and $\mathrm{SiO}(100 \mathrm{~nm})$ on silicon were also studied, which, however, show faster etching rates in the $50^{\circ} \mathrm{C} \mathrm{HF}: \mathrm{HNO}_{3}$ solution. LPCVD nitride, on the other hand, was very resistant to the $\mathrm{HF}: \mathrm{HNO}_{3}$ chemical, thereby providing an optimum masking layer for the etch.

To fabricate hemi-spherical cavities, LPCVD nitride coated silicon wafer samples were submerged in the $\mathrm{HF}: \mathrm{HNO}_{3}$ (1:9 by volume) solution for $60 \mathrm{~min}$ at room temperature. No agitation was applied during this step in order to better control the 
etching rate and surface smoothness. From our experiments, it was evident that the LPCVD nitride mask is imperative for the wet etching, in contrast with PECVD nitride or SiO. After the etching, the nitride mask was stripped off using phosphoric acid at $75^{\circ} \mathrm{C}$. Finally, the wafer was rinsed with DI water before the metallization step.

\section{Reflector Formation}

A 5- $\mu$ m-thick Parylene C layer was deposited (SCS Labcoter 2PDS 2010, Specialty Coating Systems) over the etched silicon cavity, followed by thermal evaporation of $100 \mathrm{~nm}$ aluminum (Al) (Auto306, Edwards). The deposited Al was selectively patterned using a Shipley S1813 photoresist mask and etched with Transene Aluminum Etchant A. It should be noted that this selective $\mathrm{Al}$ etching is optional, however, this step was performed to eliminate the possibility of reflection from LED side emission which might reduce the spatial resolution. Moreover, because the interface strength between large area $\mathrm{Al}$ and PDMS is not wellunderstood, the Al layer was selectively etched to ensure a good adhesion. Due to its high reflectivity, the $\mathrm{Al}$ within the cavity is expected to work as the mirror for reflecting the LED backside emission and focusing the diverged light beams.

\section{Planarization Using PDMS}

Due to the presence of the cavities, planarization using polymers was desired in order to create an even surface for fabricating interconnect wires and contact pads for LED integration. Polydimethylsiloxane (PDMS) was selected as the planarization polymer due to the ease of processing and also its lower refractive index (1.4) when compared with SU-8 (1.67). PDMS pre-polymer was prepared by mixing the elastomer base and curing agent in a weight ratio of 10:1 (Sylgard 184, Dow Corning). During planarization (Figures 2A,B), a Parylene C coated secondary wafer was spin-coated with a $10-\mu \mathrm{m}$-thick PDMS layer $(5,000$ $\mathrm{rpm}, 60 \mathrm{~s})$. Concurrently, a thin layer of PDMS was coated on top of the primary wafer where the cavities were constructed. Both the primary and secondary wafers were coated with PDMS to ensure sufficient PDMS filling in the reflector cavity and optimal adhesion. The primary wafer was then attached with the secondary wafer on the PDMS coated sides. The bonded wafers were subjected to a $9.8 \mathrm{~N}$ weight (16.33 $\mathrm{kPa}$ pressure) for $24 \mathrm{~h}$ at room temperature $\left(25^{\circ} \mathrm{C}\right)$ until the PDMS adhesive cured completely. This slow curing step allows PDMS to flow into the cavities with minimal air bubbles. After the soft curing, the bonded samples were baked in a vacuum oven at $50^{\circ} \mathrm{C}$ for $30 \mathrm{~min}$, followed by a $100^{\circ} \mathrm{C}$ bake for $5 \mathrm{~min}$. The higher temperature baking at $100^{\circ} \mathrm{C}$ ensured hardening of the PDMS layer toward further metallization and LED bonding processes. After hard baking, the secondary wafer was completely detached from the rest of the sample by delamination at the Parylenesilicon interface, resulting in the planarized Parylene-PDMSParylene structure to rest only on the primary wafer.

\section{Interconnect Metal Deposition and LED Bonding}

A $5 \mathrm{~nm} / 600 \mathrm{~nm} \mathrm{Ti} / \mathrm{Cu}$ layer was thermally evaporated, followed by UV photolithography with a Shipley S1813 photoresist mask. The photoresist mask was stripped off by rinsing the sample with acetone, IPA, and DI water. Afterward, a thin Parylene C layer $(2 \mu \mathrm{m})$ was deposited and selectively patterned to expose the bonding pads by oxygen plasma (58 sccm $\mathrm{O}_{2}, 250 \mathrm{~W}$ ). Commercially available CREE TR2227 blue LEDs were bonded onto interconnect metal pads by applying low melting point (LMP) solder (melting point at $\sim 62^{\circ} \mathrm{C}, 144$ ALLOY Field's Metal, Rotometals, Inc.) on the exposed metal contacts (Kwon et al., 2013).

\section{Packaging}

After LED assembly, the arrays were completely encapsulated by $15 \mu \mathrm{m}$ Parylene $\mathrm{C}$ to protect the devices from delamination, deformation, and corrosion due to the humidity and tissue fluids present in the in vitro and in vivo environments. The contact pads were connected to the power supply through flexible wires.

\section{Device Characterization Surface Morphology and Optical Property Measurement}

Silicon cavities were scanned in a NanoMap-500LS Surface Profilometer to quantify the horizontal and vertical etch profiles of the cavities. Inspection of surface topography was done using a Hitachi S-4700II field emission scanning electron microscope (FESEM). The surface roughness of the Al-coated reflective layer was measured using an atomic force microscope (AFM, SPM 3100 , Veeco). Light intensity for both the bare LED and reflectorcoupled devices were measured using a Newport 818-SL optical detector and Newport 843-R series optical power meter. The devices were tested under a probe station in air, and the detector was placed at a distance of $7 \mathrm{~mm}$ above the LED surface in all of the optical measurements.

\section{Thermal Property Measurement}

The temperature profile of the stimulator during continuous operation provides critical information to study the effect of the silicon reflector on LED heat dissipation. When a single LED was continuously powered by $\sim 1-25 \mathrm{~mA}$ current $(\sim 2.6-73 \mathrm{~mW})$, the thermal energy dissipation of the array was characterized in air using a thermal imaging camera (FLIR E6, FLIR ${ }^{\circledR}$ Systems, Inc) with an infrared resolution of $160 \times 120$ pixels and a temperature sensitivity of $0.06^{\circ} \mathrm{C}$. The imaging camera was calibrated by the manufacturer. The temperature of the LED surface, being the spot where the temperature would be the highest, was recorded using the imaging camera. The maximal temperature increase at the activated LED site was quantified with respect to the ambient temperature $\left(22^{\circ} \mathrm{C}\right)$ and compared to that of a bare $\mu$-LED.

\section{Simulation}

Optical intensity captured at a specific parallel plane from the LED surface was simulated using TracePro (Lambda Research Corporation). As illustrated in Figure 3a, the model used for the simulation consisted of a micro LED with the same physical dimensions as the CREE TR2227 LED $(270 \times 220 \times 50 \mu \mathrm{m})$ and a silicon cavity with a reflective layer of a standard mirror (95\%). Air was used as the surrounding medium to mimic the actual environment of device measurements. The effect of the reflective mirror on the optical intensity of the overall 


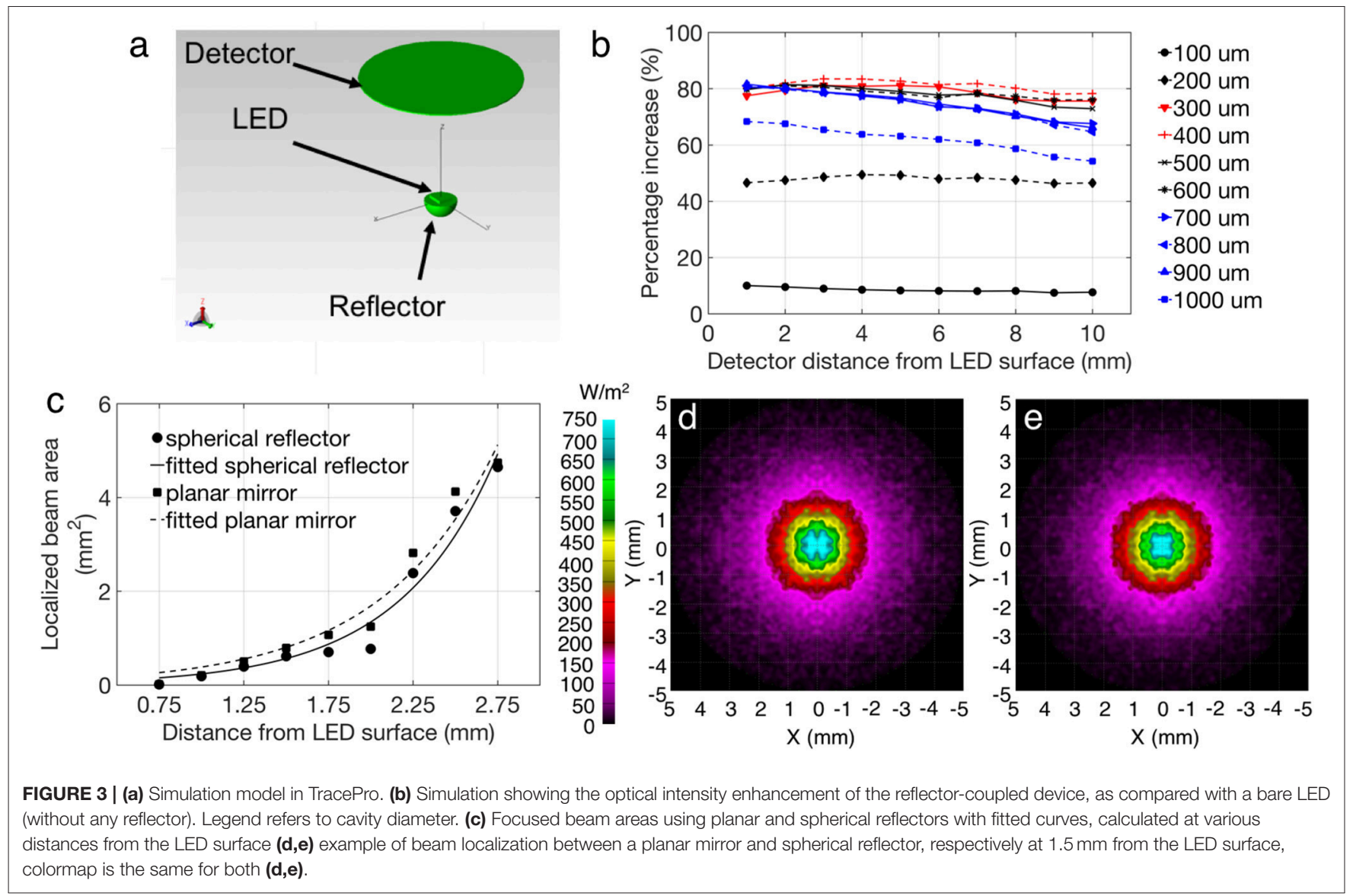

device was studied by varying the cavity diameter and the distance of the detector plane from the LED surface. For this study, the detector diameter of $10 \mathrm{~mm}$ was selected, which is consistent with the detector (Newport 818-SL) aperture used in the experimental setup. The percentage increase was calculated by normalizing the increased light intensity from the reflector-coupled LED with respect to the intensity of a bare $\mu$-LED at a certain separation: $\left(I_{\text {ref }}-I_{\text {bare }}\right) / I_{\text {bare }}$, where $I_{\text {ref }}$ and $I_{\text {bare }}$ are the simulated light intensity of reflectorcoupled and bare LEDs, respectively. Figure $\mathbf{3 b}$ shows that the intensity enhancement goes higher as the diameter of the reflector cavity increases from 100 to $400 \mu \mathrm{m}$, and then drops gradually as the cavity diameter continuously increases. For a $300 \mu \mathrm{m}$ diameter reflector, an intensity enhancement of $78 \%$ was achieved, when compared to a bare LED without a reflector. A better localization of light beams was observed when the hemi-spherical cavity was compared with a planar mirror in simulation. Figure 3c shows the quantitative analysis of centralized illumination area using spherical reflector over a planar mirror calculated at different distances from the LED surface, while Figures 3d,e show the examples of simulated light spots at the same separation in the same intensity range. The center spot beam localization was estimated by calculating the central area of the beam (from the planar mirror or hemispherical reflector) at the same intensity level and at the same distance from the detector. Fitted curves have been included in Figure $3 c$ to indicate the trend of center beam localization for the mentioned geometries, and shows an advantage of the hemi-spherical cavity reflector over a planar mirror, in terms of localized beam area reduction. This advantage will enable better spatial resolution of optical stimulation, which is desired for optogenetics applications.

\section{In vitro Experiments}

To establish proof-of-principle for application with an optogenetic system, we assessed the efficacy of the $\mu$-LEDs to activate an optically-induced gene expression system (with and without reflectors). Successful induction is confirmed by the visual identification of the expression of a red fluorescent reporter following genetic modification and exposure to blue light (Figure 6A). For these in vitro experiments, human embryonic kidney cells (HEK 293) were used. HEK 293 were maintained under Dulbecco's modified Eagle's medium (DMEM; Life Technologies) supplemented with 10\% fetal bovine serum (FBS; Sigma) and $1 \%$ penicillin/streptomycin (Life Technologies). Colonies were passaged every 3-4 days by chemical dissociation utilizing TrypLE (Life Technologies). Cells were kept in an incubator at $37^{\circ} \mathrm{C}$ with $5 \% \mathrm{CO}_{2}$. Prior to light induction experiments, cells were passaged onto 24-well plates at 68,000 cells/well. Cells were then transiently transfected 

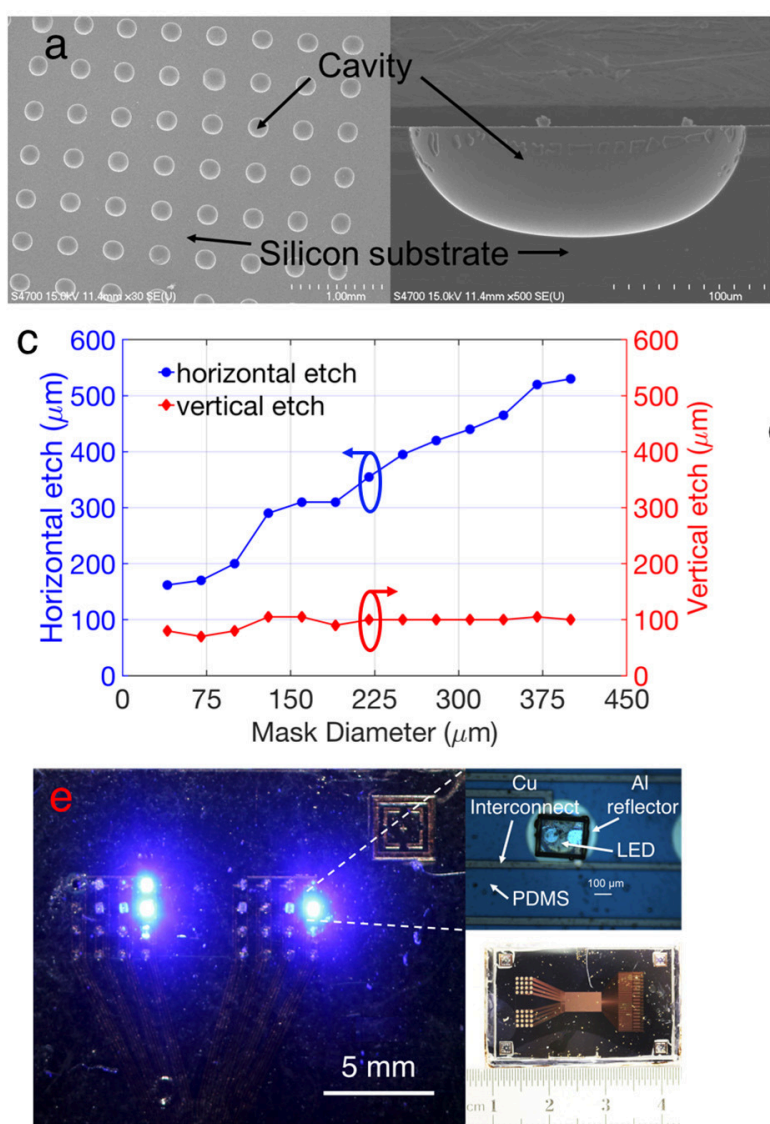

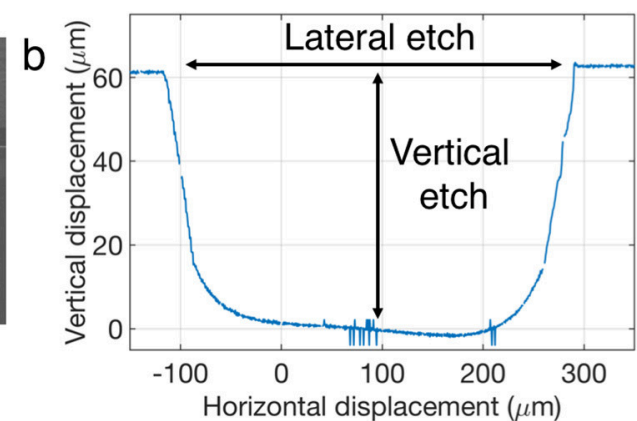

d

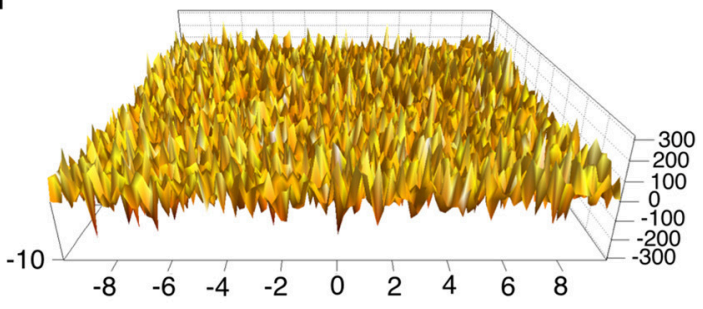

f

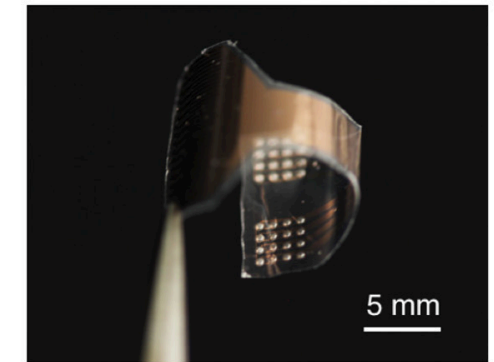

FIGURE 4 | (a) SEM images of the etched silicon cavity. (b) Surface profilometer results for calculating the etched lateral and vertical distances. (c) The etched distance with respect to mask diameter. (d) AFM image showing the smoothness of the etched cavity ( $x$ axis units in $\mu \mathrm{m}, y$ axis units in nm). (e) A fabricated array stimulator, coupled with the cavity reflector. (f) A released stimulator array before LED bonding.

utilizing Lipofectamine 3000 (Invitrogen) by following the manufacturer's protocol. Each well was transfected with 200 ng of DNA including 40 ng of activator plasmid ["EL222," a blue light-activated gene expression system; (Motta-Mena et al., 2014; Winter et al., 2017)], 20 ng of reporter gene (red fluorescence protein, RFP) and $140 \mathrm{ng}$ of PUC (an empty plasmid). Twelve (12) to sixteen (16) hours after DNA transfection, cells were exposed to blue light $(465 \mathrm{~nm})$ from LEDs for either 240 or $480 \mathrm{~min}$. Two wells were exposed to standard (bare) LEDs, two wells were exposed to reflectorcoupled LEDs, and four were kept under dark conditions as a control.

After light stimulation, cells were maintained in the incubator. After $72 \mathrm{~h}$, each well was imaged using a fluorescent microscope (Leica MZ10 F) to assess optical induction of the RFP reporter construct. All plasmids used in this study were provided by BiomiLab, LLC (Winter et al., 2017). Individual images received manual threshold utilizing Ocular software (QImaging) to eliminate background fluorescence. Images from all wells were further analyzed with an in-house MATLAB script to calculate the number of "zero" pixels (where a fluorescent signal was not detected) and non-zero pixels (where fluorescent signal was detected) to obtain the percentage of pixels above threshold (number of non-zero pixels divided by total number of pixels, $\%$ PAT, multiplied by a factor of 10,000 to facilitate comparison, Table 1) for each image. Lastly, an additional MATLAB script (Kozai et al., 2014; Salatino et al., 2017) was used to assess the spatial gradient of gene expression in a preliminary test by creating bins of intensity in area increments (two wells were exposed to light for $480 \mathrm{~min}$ : one with the bare LED stimulator and the other with the reflector coupled stimulator). To assess images spatially, bins were utilized to quantify mean intensity as a function of distance from the center of the LED location. Twelve 200 micron-width bins were analyzed which projected radially from the center of the LED site. Location of the center was manually selected to accurately represent the center of the region that received direct light exposure. The LEDs were manually positioned at the center on each well, based on visual inspection. The manual procedure for LED placement, as well as the flexibility of the LEDs, likely contributed to a slightly off-center placement relative to the center of the well (Figure 6A). 
TABLE 1 | Average \%PAT (multiplied by a factor of 10,000 to facilitate comparison) for control, bare LED stimulator and reflector coupled stimulator.

\begin{tabular}{lll}
\hline Average \%PAT per condition & $\mathbf{2 4 0} \mathbf{m i n}(\boldsymbol{n}=\mathbf{2})$ & $\mathbf{4 8 0} \mathbf{m i n}(\boldsymbol{n}=\mathbf{1})$ \\
\hline Control (no light) & 6.86 & 17.21 \\
Bare LED stimulator & 12.12 & 83.41 \\
Reflector coupled stimulator & 17.37 & 88.39 \\
\hline
\end{tabular}

\section{RESULTS AND DISCUSSIONS}

\section{Fabricated Devices}

Figure 4a provides SEM images showing the morphology of the silicon cavity after wet etching. The cavity formation is the most important step for the reflector fabrication, and the surface morphology of the cavities need to be comparatively smooth to ensure efficient light reflection with minimum scattering. AFM analysis in Figure $\mathbf{4 b}$ shows a small mean roughness of $\sim 72 \mathrm{~nm}$, confirming the smooth surface of the etched cavity. The horizontal and vertical etching rates of silicon cavities were calculated based on the profilometry data, as shown in Figure 4c. The results indicate that the etching rates in the horizontal and the vertical direction are not the same, with the opening of the nitride mask being a limiting factor. Due to the lack of agitation, the vertical etching rate is slower than the horizontal rate and less dependent on the size of nitride opening (Figure 4d). This phenomenon forced the cavities not to be perfectly hemispherical, thereby creating a deviation between the theoretical concept and the realized cavity geometry. Consequently, a deviation should also be expected between the simulation results (which considers the cavity to be perfectly hemi-spherical) and the experimental results. Figure 4e illustrates a fabricated 32channel stimulator array on a silicon chip, with illuminated LEDs on both panels. There is a slight misalignment between the LED bonding pads and cavity, due to the inaccuracy of the photolithography process. Deposition of the Parylene C layer allows the release of the array from the silicon substrate and transform it to a fully flexible free-standing stimulator array to adapt with corrugated brain surfaces by conformal contact. Figure $4 \mathbf{f}$ shows a released array from the substrate wafer, where the bending is due to the mechanical stress of the thin Parylene C layer after release, and could be reduced by stabilizing and flattening the array on a separate substrate using water as adhesive.

\section{Optical Properties}

Figure 5A shows the optical intensity enhancement due to the coupled reflector, which was measured experimentally with respect to a bare (no-reflector-coupled) optical stimulator. The reflector-coupled devices achieved the minimal and maximal enhancement of 49 and 65\%, respectively, verifying the effectiveness of the reflector to collect the rear surface emission of the LED. For these benchtop experiments, the LEDs were coupled with 300- $\mu \mathrm{m}$-diameter reflectors that ensure the optimized intensity enhancement as suggested by the simulation above. The experimental data was slightly lower than simulated values, which could be attributed to several factors: fabrication imperfections, oval-shaped cavity profile, inaccuracy of manual alignment between the LED and reflector layers, and high contact impedance of the LED-solder-contact interface. Regarding in-vivo experiments, a common excitatory opsin is channelrhodopsin (ChR2) that can be activated by blue light with threshold of $1 \mathrm{~mW} / \mathrm{mm}^{2}$ (Aravanis et al., 2007). Preliminary benchtop measurements indicate that our intensity enhanced stimulators surpass this threshold even at a low applied current of $<2.5 \mathrm{~mA}$. It is of note that the detector and LED surface had a separation of $7 \mathrm{~mm}$ due to the use of a probe station. The measured intensity might be further improved if the separation could be reduced by improving the measurement setup.

\section{Thermal Properties}

Although the brain is only $2 \%$ of the body's mass, $20 \%$ of the cardiac output is delivered toward it, indicating a high metabolic demand in the brain (Wolf, 2008). A high resting blood flow clears the heat generated by the brain. Adverse effects of increased temperature due to implants, on top of this high metabolism activity have been observed in the central nervous system (CNS) where breakdown of blood-brain barrier was observed after $60 \mathrm{~min}$ at $42^{\circ} \mathrm{C}$ along with some neuronal death (6\%) after $60 \mathrm{~min}$ of heating at $40.5^{\circ} \mathrm{C}$ (Goldstein et al., 2003). A maximum tolerable heat dose of $42.0-42.5^{\circ} \mathrm{C}$ for $40-60 \mathrm{~min}$ or $43^{\circ} \mathrm{C}$ for $10-30 \mathrm{~min}$ (Haveman et al., 2005) was established after a review study on regional exposure of the animal brain and spinal cord. The American Association of Medical Instrumentations (ANSI/AAMI) has a standard limit of $2^{\circ} \mathrm{C}$ increase for chronic biomedical implants for neuro-stimulators (ISO-14708-1). A change in temperature was calculated by subtracting the initial temperature $\left(\sim 22^{\circ} \mathrm{C}\right.$ when the LED was off) from the operating temperature of the LED measured by the infrared camera. A temperature increase of $<1^{\circ} \mathrm{C}$ was observed when our stimulators were operated under continuous current inputs (Figure 5B), even at higher light intensities ( $>8$ $\mathrm{mW} / \mathrm{mm}^{2}$ ). It could be noted that when compared with bare LEDs, the reflector-coupled stimulators dissipate less heat at $>8$ $\mathrm{mW} / \mathrm{mm}^{2}$ intensities, potentially due to the decreased thermal conductivities of silicon and PDMS at higher temperatures (Silicon Wafer - Thermophysical Properties, n.d).

\section{In vitro Results}

In vitro tests confirmed proof-of-principle for the successful induction of red fluorescent reporter expression with $\mu$-LEDs, as well as a more robust and spatially localized area of induction with the use of reflectors (Figure 6; Table 1). Fluorescent microscope images of each well were analyzed for either \%PAT or intensity (see Methods) at each of the various duration settings chosen for light exposure. Qualitatively, light-induced gene expression was visually observable following $240 \mathrm{~min}$ of exposure (Figure 6A). Table 1 shows \%PAT values obtained from our first in-house MATLAB script and represents average values of \%PAT for two images per condition $(n=2$, where the entire image area is quantified to deliver a single output value) for a blue-light exposure duration of $240 \mathrm{~min}$ and \%PAT values for one image per condition $(n=1)$ for a blue-light 

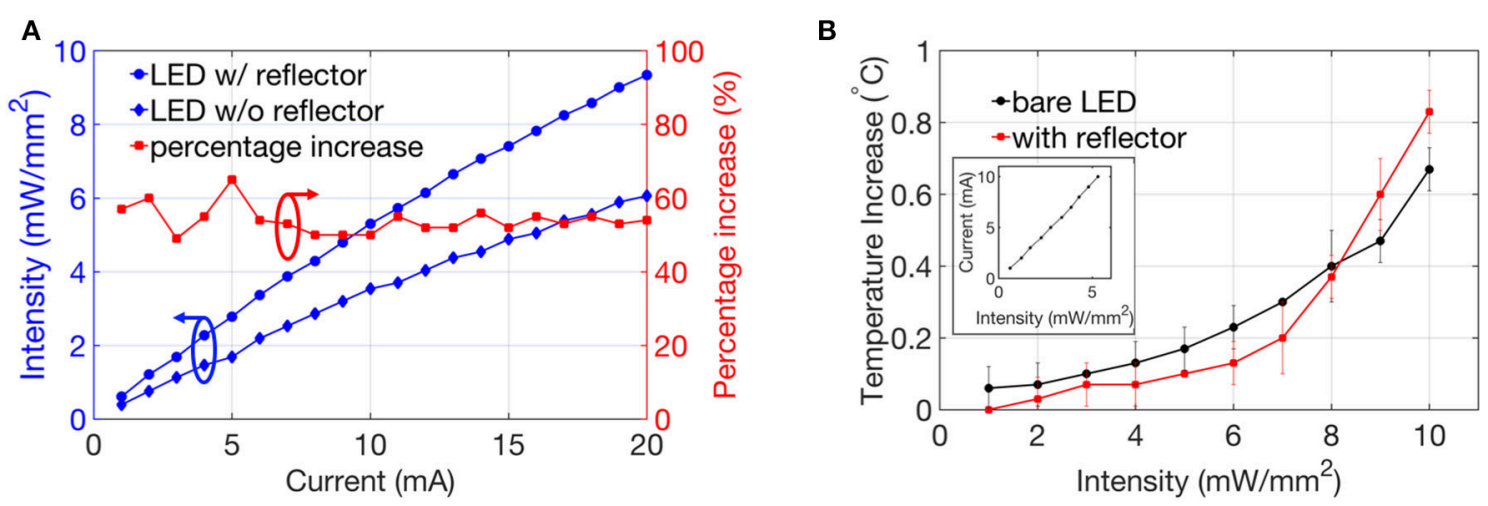

FIGURE 5 | (A) Optical intensity and (B) temperature increase compared with a bare micro LED stimulator (in inset-correlation of intensity vs. current for reflector coupled stimulators).
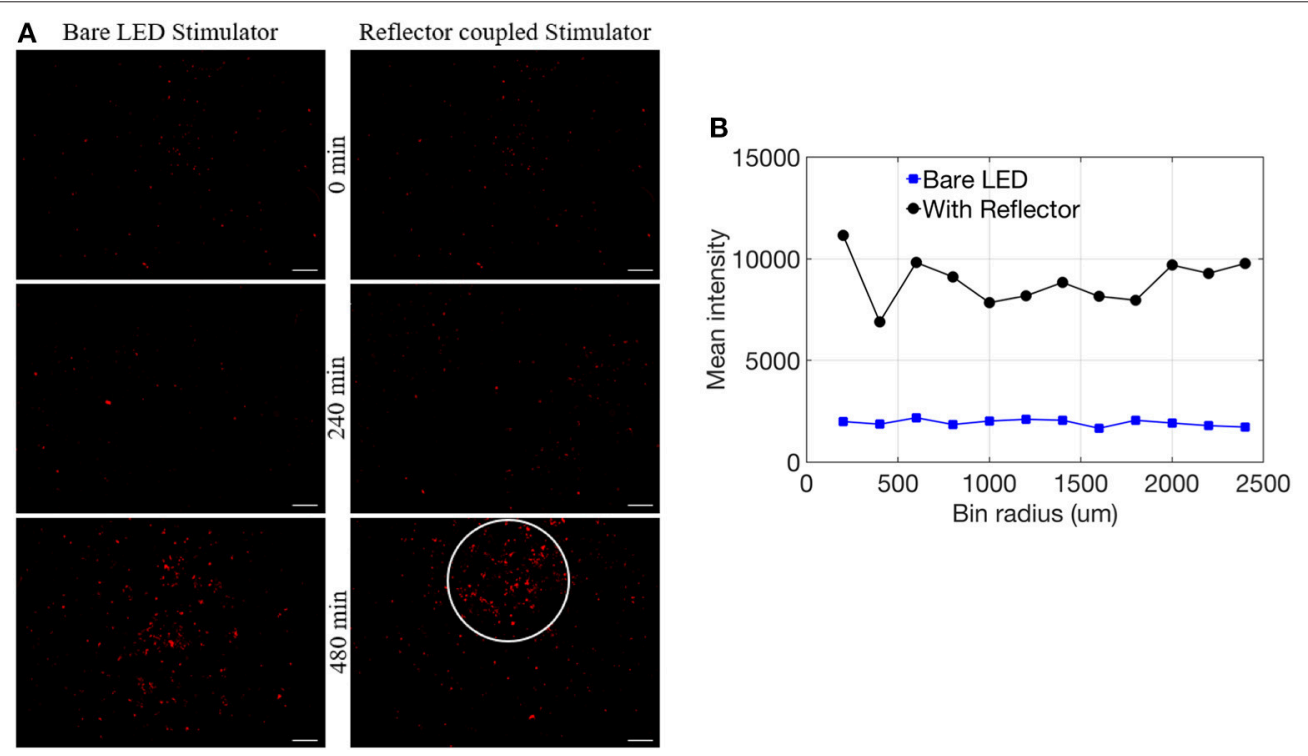

FIGURE 6 | (A) Comparison of bare LED stimulator and reflector coupled stimulator fluorescence of RFP reporter for 0, 240 , and 480 min (scale = 1 mm). (B) Mean intensity data with respect to bin radius $(n=1)$.

exposure duration of $480 \mathrm{~min}$. As expected, light-stimulated wells registered higher \%PAT values than non-exposed wells, verifying successful optical induction of the reporter gene (Table 1). Due to the residual dark-state binding of the activator, some fluorescence in dark conditions was observed and served as a baseline for quantification. At $480 \mathrm{~min}$, higher \%PAT values were measured for the cells exposed to both the reflector coupled LEDs and for the bare LEDs when compared to the $240 \mathrm{~min}$ exposure duration (Table 1). Additionally, reflectorcoupled LEDs registered slightly elevated \%PAT values for both timepoints (Table 1; values multiplied by 10,000 to facilitate detection of differences between values).

Utilizing our second MATLAB script, preliminary observations suggest a more spatially localized pattern of reporter expression at $480 \mathrm{~min}$ (white circle in Figure 6A) potentially related to focused illumination enabled by the reflectors. To analyze the expression pattern as a function of distance from the center of induction, mean intensity per bin was calculated and normalized by using the corners of each image. This confirmed elevated expression in comparison to standard LEDs within the highlighted region of interest (Figure 6B). Future work will need to confirm this result with an expanded data set.

\section{CONCLUSION}

In this paper, we have designed, fabricated, simulated and characterized a multichannel, reflector-coupled $\mu$-LED array for optogenetic experiments. A wafer-level fabrication method was implemented to monolithically integrate multichannel reflectors with $\mu$-LEDs. Our experimental results demonstrate that using the Al-coated cavity as a back reflector enables 
significant enhancement in light intensity by at least $49 \%$ and a maximum of $65 \%$ in comparison to a bare $\mu$-LED. Simulation using optical ray tracing verified the effectiveness of the reflector to improve the output light intensity, while also providing a guideline toward future optimization of device design. Additionally, our 32-channel stimulator array allows high-resolution spatial stimulation, enabling multi-site stimulation for localized neuronal activity analysis. Furthermore, the structural and packaging material used in our device is Parylene $\mathrm{C}$, which ensures the biocompatibility and reliability of the stimulators in the brain fluids. When compared with optical fiber based stimulators, our proposed array is advantageous in terms of multi-site stimulation and motivates toward an untethered wireless device. In addition, our array demonstrated improved heat dissipation $\left(<1^{\circ} \mathrm{C}\right)$, compared to an optical fiber based stimulator reported by $\mathrm{Y}$. Shin et al. where a temperature increase of $1-1.5^{\circ} \mathrm{C}$ was observed (Shin et al., 2016). Finally, the effectiveness of the reflectorcoupled LEDs for localized light induction of optogenetic DNA was validated in vitro using human embryonic kidney cells.

\section{REFERENCES}

Abaya, T. V. F., Blair, S., Tathireddy, P., Rieth, L., and Solzbacher, F. (2012). A 3D glass optrode array for optical neural stimulation. Biomed. Opt. Express 3, 3087-3104. doi: 10.1364/BOE.3.003087

Adamantidis, A. R., Zhang, F., Aravanis, A. M., Deisseroth, K., and de Lecea, L. (2007). Neural substrates of awakening probed with optogenetic control of hypocretin neurons. Nature 450: 420. doi: 10.1038/nature06310

Albero, J., Nieradko, L., Gorecki, C., Ottevaere, H., Gomez, V., Thienpont, H., et al. (2009). Fabrication of spherical microlenses by a combination of isotropic wet etching of silicon and molding techniques. Opt. Express 17, 6283-6292. doi: 10.1364/OE.17.006283

Aldaoud, A., Soto-Breceda, A., Tong, W., Conductier, G., Tonta M. A., Coleman, H. A., et al. (2018). Wireless multichannel optogenetic stimulators enabled by narrow bandwidth resonant tank circuits. Sens. Actuators A Phys. 271, 201-211. doi: 10.1016/j.sna.2017.12.051

Aravanis, A. M., Wang, L. P., Zhang, F., Meltzer, L. A., Mogri, M. Z., Schneider, B. M., et al. (2007). An optical neural interface: in vivo control of rodent motor cortex with integrated fiberoptic and optogenetic technology. J. Neural Eng. 4: S143. doi: 10.1088/1741-2560/4/3/S02

Ayling, O. G., Harrison, T. C., Boyd, J. D., Goroshkov, A., and Murphy, T. H. (2009). Automated light-based mapping of motor cortex by photoactivation of channelrhodopsin-2 transgenic mice. Nat. Methods 6: 219. doi: $10.1038 /$ nmeth.1303

Bi, X., Xie, T., Fan, B., Khan, W., Guo, Y., and Li, W. (2016). A flexible, micro-lenscoupled LED stimulator for optical neuromodulation. IEEE Trans. Biomed. Circuits Syst. 10, 972-978. doi: 10.1109/TBCAS.2016.2599406

Biswas, D. K., Sinclair, M., Hyde, J., and Mahbub, I. (2018). “An NFC (nearField Communication) based wireless power transfer system design with miniaturized receiver coil for optogenetic implants," in 2018 Texas Symposium on Wireless and Microwave Circuits and Systems (WMCS) (Waco, TX: IEEE), $1-5$.

Campagnola, L., Wang, H., and Zylka, M. J. (2008). Fiber-coupled light-emitting diode for localized photostimulation of neurons expressing channelrhodopsin2. J. Neurosci. Methods 169, 27-33. doi: 10.1016/j.jneumeth.2007.11.012

Chen, S., Pei, W., Gui, Q., Chen, Y., Zhao, S., Wang, H., et al. (2013). A fiber-based implantable multi-optrode array with contiguous optical and electrical sites. J. Neural Eng. 10:046020. doi: 10.1088/1741-2560/10/4/046020

Deisseroth, K. (2011). Optogenetics. Nat. Methods 8, 26-29. doi: $10.1038 /$ nmeth.f.324

\section{AUTHOR CONTRIBUTIONS}

Unless otherwise stated, WK fabricated the devices, performed the benchtop experiments and simulations and wrote the manuscript. MS performed the in vitro experiments and analysis, wrote sections in vitro Experiments and in vitro Results, created the Figure 6. EP and WL supervised and advised on the concept, design, and experiments. All authors contributed to manuscript revision, read and approved the submitted version.

\section{FUNDING}

This work was supported in part by the National Science Foundation under the Award Numbers ECCS-1407880 and Michigan State University.

\section{ACKNOWLEDGMENTS}

The authors would also like to thank BiomiLab, LLC for providing the plasmids for this study and Bailey Winter for providing the Matlab script to analyze the pixel density.

Fan, B., Kwon, K. Y., Weber, A. J., and Li, W. (2014). "An implantable, miniaturized SU-8 optical probe for optogenetics-based deep brain stimulation," in 2014 36th Annual International Conference of the IEEE Engineering in Medicine and Biology Society (Chicago, IL: IEEE), 450-453. doi: 10.1109/EMBC.2014.6943625

Gagnon-Turcotte, G., Maghsoudloo, E., Messaddeq, Y., De Koninck, Y., and Gosselin, B. (2017). "A wireless photostimulator for optogenetics with live animals," in New Circuits and Systems Conference (NEWCAS), 2017 15th IEEE International (Strasbourg: IEEE), 193-196.

Goldstein, L. S., Dewhirst, M. W., Repacholi, M., and Kheifets, L. (2003). Summary, conclusions and recommendations: adverse temperature levels in the human body. Int. J. Hyperthermia 19, 373-384. doi: 10.1080/0265673031000090701

Grosenick, L., Marshel, J. H., and Deisseroth, K. (2015). Closedloop and activity-guided optogenetic control. Neuron 86, 106-139. doi: 10.1016/j.neuron.2015.03.034

Grossman, N., Poher, V., Grubb, M. S., Kennedy, G. T., Nikolic, K., McGovern, B., et al. (2010). Multi-site optical excitation using ChR2 and micro-LED array. J. Neural Eng. 7:016004. doi: 10.1088/1741-2560/7/1/016004

Han, X., Qian, X., Bernstein, J. G., Zhou, H. H., Franzesi, G. T., Stern, P., et al. (2009). Millisecond-timescale optical control of neural dynamics in the nonhuman primate brain. Neuron 62, 191-198. doi: 10.1016/j.neuron.2009.03.011

Haveman, J., Sminia, P., Wondergem, J., van der Zee, J., and Hulshof, M. C. (2005). Effects of hyperthermia on the central nervous system: what was learnt from animal studies? Int. J. Hyperthermia 21, 473-487. doi: 10.1080/02656730500159079

Jeong, J. W., McCall, J. G., Shin, G., Zhang, Y., Al-Hasani, R., Kim, M., et al. (2015). Wireless optofluidic systems for programmable in vivo pharmacology and optogenetics. Cell 162, 662-674. doi: 10.1016/j.cell.2015.06.058

Khan, W., Jia, Y., Madi, F., Weber, A., Ghovanloo, M., and Li, W. (2018). "A miniaturized, wirelessly-powered, reflector-coupled single channel opto neurostimulator," in 2018 IEEE Micro Electro Mechanical Systems (MEMS) (Belfast: IEEE), 174-177. doi: 10.1109/MEMSYS.2018. 8346512

Khan, W., and Li, W. (2017). "Wafer level fabrication method of hemispherical reflector coupled micro-led array stimulator for optogenetics," in 2017 19th International Conference on Solid-State Sensors, Actuators and Microsystems (TRANSDUCERS) (Kaohsiung: IEEE), 2231-2234.

Kim, T. I., McCall, J. G., Jung, Y. H., Huang, X., Siuda, E. R., Li, Y., et al. (2013). Injectable, cellular-scale optoelectronics with applications 
for wireless optogenetics. Science 340, 211-216. doi: 10.1126/science.12 32437

Kozai, T. D., Gugel, Z., Li, X., Gilgunn, P. J., Khilwani, R., Ozdoganlar, O. B., et al. (2014). Chronic tissue response to carboxymethyl cellulose based dissolvable insertion needle for ultra-small neural probes. Biomaterials 35, 9255-9268. doi: 10.1016/j.biomaterials.2014.07.039

Kwon, K., and Li, W. (2013). "Integrated multi-LED array with three-dimensional polymer waveguide for optogenetics," in 2013 IEEE 26th International Conference on Micro Electro Mechanical Systems (MEMS) (Taipei: IEEE), 1017-1020.

Kwon, K. Y., Sirowatka, B., Weber, A., and Li, W. (2013). Opto- $\mu$ ECoG array: a hybrid neural interface with transparent $\mu$-ECoG electrode array and integrated LEDs for optogenetics. IEEE Trans. Biomed. Circuits Syst. 7, 593-600. doi: 10.1109/TBCAS.2013.2282318

McAlinden, N., Gu, E., Dawson, M. D., Sakata, S., and Mathieson, K. (2015). Optogenetic activation of neocortical neurons in vivo with a sapphire-based micro-scale LED probe. Front. Neural Circuits 9:25. doi: 10.3389/fncir.2015.00025.

McGovern, B., Berlinguer Palmini, R., Grossman, N., Drakakis, E. M., Poher, V., Neil, M. A., et al. (2010). A new individually addressable micro-LED array for photogenetic neural stimulation. IEEE Trans. Biomed. Circuits Syst. 4, 469-476. doi: 10.1109/TBCAS.2010.2081988

Montgomery, K. L, Yeh, A. J., Ho, J. S., Tsao, V., Mohan Iyer, S., Grosenick, L., et al. (2015). Wirelessly powered, fully internal optogenetics for brain, spinal and peripheral circuits in mice. Nat. Methods 12, 969-974. doi: 10.1038/nmeth. 3536

Motta-Mena, L. B., Reade, A., Mallory, M. J., Glantz, S., Weiner, O. D., Lynch, K. W., et al. (2014). An optogenetic gene expression system with rapid activation and deactivation kinetics. Nat. Chem. Biol. 10, 196-202. doi: $10.1038 /$ nchembio. 1430

Nussinovitch, U., and Gepstein, L. (2015). Optogenetics for in vivo cardiac pacing and resynchronization therapies. Nat. Biotechnol. 33: 750. doi: 10.1038/nbt.3268

Park, S. I., Brenner, D. S., Shin, G., Morgan, C. D., Copits, B. A., Chung, H. U., et al. (2015). Soft, stretchable, fully implantable miniaturized optoelectronic systems for wireless optogenetics. Nat. Biotechnol. 33, 1280-1286. doi: 10.1038/ nbt. 3415

Salatino, J. W., Ludwig, K. A., Kozai, T. D. Y., and Purcell, E. K. (2017). Glial responses to implanted electrodes in the brain. Nat. Biomed. Eng. 1:862. doi: 10.1038/s41551-017-0154-1

Shin, G., Gomez, A. M., Al-Hasani, R., Jeong, Y. R., Kim, J., Xie, Z., et al. (2017). Flexible near-field wireless optoelectronics as subdermal implants for broad applications in optogenetics. Neuron 93, 509-521.e3. doi: 10.1016/j.neuron.2016.12.031

Shin, Y., Yoo, M., Kim, H. S., Nam, S. K., Kim, H. I., Lee, S. K., et al. (2016). Characterization of fiber-optic light delivery and light-induced temperature changes in a rodent brain for precise optogenetic neuromodulation. Biomed. Opt. Express 7, 4450-4471. doi: 10.1364/BOE.7.004450
Silicon Wafer - Thermophysical Properties. (n.d). NETZSCH Analyzing and Testing. Available online at: https://www.netzsch-thermal-analysis. com/us/materials-applications/photovoltaics/silicon-wafer-thermophysicalproperties/ (Accessed July 31, 2018).

Stark, E., Koos, T., and Buzsáki, G. (2012). Diode probes for spatiotemporal optical control of multiple neurons in freely moving animals. J. Neurophysiol. 108, 349-363. doi: 10.1152/jn.00153.2012

Tokuda, T., Kimura, H., Miyatani, T., Maezawa, Y., Kobayashi, T., Noda, T., et al. (2012). CMOS on-chip bio-imaging sensor with integrated micro light source array for optogenetics. Electron. Lett. 48, 312-314. doi: 10.1049/el.2011.4087

Williams, K. R., Gupta, K., and Wasilik, M. (2003). Etch rates for micromachining processing-part II. J. Microelectromech. Syst. 12, 761-778. doi: 10.1109/JMEMS.2003.820936

Winter, B. M., Setien, M. B., Salatino, J. W., Blanke, N., Thompson, C. H., Smith, K. R., et al. (2017). "Control of cell fate and excitability at the neural electrode interface: genetic reprogramming and optical induction," in Life Sciences Conference (LSC), 2017 IEEE (Sydney, NSW: IEEE), 157-161.

Wolf, P. D. (2008). "Thermal considerations for the design of an implanted cortical brain-machine interface (BMI)," in Indwelling Neural Implants: Strategies for Contending With the in vivo Environment, ed W. M. Reichert (Boca Raton, FL: CRC Press; Taylor \& Francis).

Wu, F., Stark, E., Im, M., Cho, I.-J., Yoon, E.-S., Buzsáki, G., et al. (2013). An implantable neural probe with monolithically integrated dielectric waveguide and recording electrodes for optogenetics applications. J. Neural Eng. 10:056012. doi: 10.1088/1741-2560/10/5/056012

Yizhar, O., Fenno, L. E., Davidson, T. J., Mogri, M., and Deisseroth, K. (2011). Optogenetics in neural systems. Neuron 71, 9-34. doi: 10.1016/j.neuron.2011.06.004

Zhang, F., Gradinaru, V., Adamantidis, A. R., Durand, R., Airan, R. D. de Lecea, L., et al. (2010). Optogenetic interrogation of neural circuits: technology for probing mammalian brain structures. Nat. Protoc. 5, 439-456. doi: $10.1038 /$ nprot.2009.226

Zorzos, A. N., Scholvin, J., Boyden, E. S., and Fonstad, C. G. (2012). Threedimensional multiwaveguide probe array for light delivery to distributed brain circuits. Opt. Lett. 37, 4841-4843. doi: 10.1364/OL.37.004841

Conflict of Interest Statement: The authors declare that the research was conducted in the absence of any commercial or financial relationships that could be construed as a potential conflict of interest.

Copyright (c) 2018 Khan, Setien, Purcell and Li. This is an open-access article distributed under the terms of the Creative Commons Attribution License (CC BY). The use, distribution or reproduction in other forums is permitted, provided the original author(s) and the copyright owner(s) are credited and that the original publication in this journal is cited, in accordance with accepted academic practice. No use, distribution or reproduction is permitted which does not comply with these terms. 